\title{
High-Resolution Microendoscopy
}

National Cancer Institute

\section{Source}

National Cancer Institute. High-Resolution Microendoscopy. NCI Thesaurus. Code C116480.

A low-cost, high-resolution imaging tool consisting of a 1-mm diameter fiber-optic bundle that produces subcellular imaging at 1,000x magnification and 4 micrometer resolution, allowing for minimally invasive bioimaging. 